\title{
SPATIAL INTERPOLATION OF
} NATURAL RADIATION LEVELS WITH PRIOR INFORMATION USING BACKPROPAGATION ARTIFICIAL NEURAL NETWORKS

\author{
J. P. Rigol-Sanchez \\ Department of Geology, University of Jaen, Spain
}

\begin{abstract}
We propose artificial neural networks (ANNs) as a tool for automatic mapping of daily observations of environmental data. A feed-forward back-propagation neural network for estimating daily natural radiation measurements at unsampled locations using prior information was developed. Feed-forward back-propagation networks were trained to learn: (a) the relationship between daily measurements and their spatial coordinates, and (b) the relationship between daily measurements made at one site and measurements made at the six surrounding closest sites. Results of the study indicate that ANNs can be used for automatic mapping of environmental (background) data with moderate success. ANN models for spatial interpolation can successfully incorporate prior information into the estimation process. However, the ANN approach to automatic mapping of environmental data presented here was clearly inappropriate for dealing with outliers. Results obtained suggest that developing two different models for estimating background values and extreme values, respectively, might be a potentially more successful approach to automatic mapping of environmental data.
\end{abstract}

\section{INTRODUCTION}

A wide variety of globally (e.g. splines, trend surfaces) and locally (e.g. inverse distance weighting, ordinary kriging) computed spatial interpolation techniques are in common use. Some methods allow to incorporate valuable information both from surrounding observations and from other covariates into the estimation process (e.g. partial thin plate splines, universal kriging, cokriging). However, the modelling process may become very cumbersome to apply when the analyst tries to incorporate more than one independent variable (i.e. covariate) into a single estimation process when using widely used methods such as thin plate splines, universal kriging or cokriging. More commonly, the relationship between the variable of interest and covariates (including spatial coordinates) may be computed using multiple linear regression or neural networks, and then the residuals are interpolated using a geostatistical method such as ordinary kriging (e.g., residual kriging) (Holdaway 1996; Demyanov et al. 2001). These processes are combined when using partial thin plate splines (Hutchinson 1995). 
Spatial interpolation using conventional methods is customarily performed adjusting model parameters using the data at hand. Parameters are re-evaluated when presented with a new dataset for the same geographical domain. This situation typically takes place when spatially interpolating daily, monthly, or yearly observations of environmental data. A spatial model must be developed for each dataset at each time step (e.g. a variogram for each month). Automatic mapping of environmental variables requires a method able to make use of prior data to adjust spatial model parameters and to generate spatial estimations when presented with new (temporally lagged) data from the same geographical domain. If spatial data follows a temporal sequence time-series forecasting techniques might ideally be incorporated into the procedure. Unlike other methods, Artificial Neural Networks (ANNs) are particularly adept at coping with non-normal and inter-correlated inputs, and managing the incorporation of additional data and expert knowledge about a particular geographical domain within the estimation process (Rigol et al. 2001). This suggests the possibility that ANNs might provide benefits over more traditional interpolation methods (Dubois and Shibli 2003). Artificial Neural Networks (ANNs) are increasingly used as spatial interpolation tools for environmental data (e.g., Pariente and Laurini 1993; Rizzo and Dougherty 1994; Pariente 1994; De Bollivier et al. 1997; Luk et al. 2000; Snell et al. 2000; Rigol et al. 2001; Antonic et al. 2001; Bryan and Adams 2002).

We propose here ANNs as a tool for automatic mapping of daily observations of environmental data. A feed-forward back-propagation neural network for estimating daily natural radiation measurements at unsampled locations using prior information was developed. The underlying idea was to extend global neural spatial regression incorporating local information from neighbour (in space and time) sites. The main objective was to train a network to learn the functional relationship between daily observations and neighbourhood observations for the corresponding day (local spatial regression) along with the potential relationship between spatial location and observed value (global spatial regression).

\section{METHODOLOGY}

\section{ARTIFICIAL NEURAL NETWORK OVERVIEW}

In their most general form, ANNs are structures inspired by biological systems, usually implemented in software (Haykin 1994). Several kinds of ANNs have been devised, being the multi-layer perceptron trained with the back-propagation algorithm the most widely used model in environmental applications. The following outline focuses on this kind of network, the architecture used for this study.

ANNs are data-processing systems that can be used as flexible regression and classification tools. A network consists of a set of basic processing elements, called units or neurons, interconnected by weighted links (Figure 1). A unit in the network sums the weighted inputs from the links feeding into it, and performs a linear or, more typically, a non-linear function on the summed value. The output is then fed to other units linked to it. During the training of a feed-forward network the weights of the network are adjusted in a process called back-propagation so as to minimise a measure of the difference between the actual output of the net and the desired output (Rumelhart et al. 1986). Thus, training or learning corresponds to the parameter estimation phase in traditional statistical models. Figure 1 illustrates a single processing unit and the architecture of a simple 
network configuration, termed multi-layer feed-forward. In this network architecture, units are arranged in layers and connected so that the information flows unidirectionally from input units, through units in one (or more) hidden layers to units in an output layer. On the other hand, error signals originate at an output neuron and flow backward through the network during the training phase. In practice, training of a network by back-propagation involves three phases (Sarle 1999): (a) the feed-forward pass of the input pattern or case, (b) the calculation of the corresponding error at the output units comparing the estimated value and the observed value or target and its back-propagation to previous layers, and (c) the adjustment of the weights. After training, application of the net (i.e. estimation, classification) involves only the first step above.

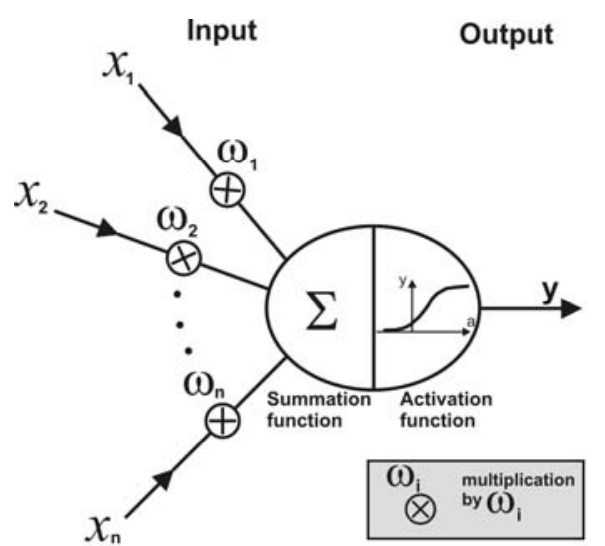

A

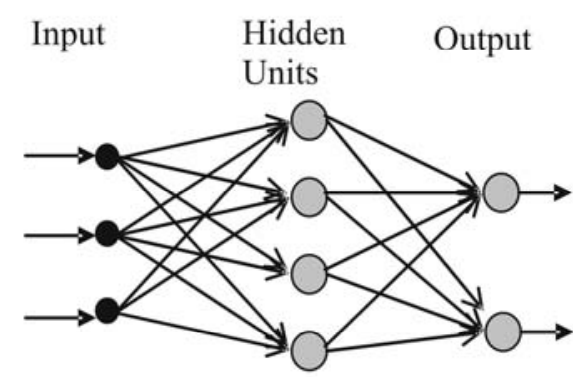

B

Figure 1

(a) Processing unit or neuron (input bias $x_{0}$ not shown), and (b) architecture of a feed-forward multi-layer network with 3 (pseudo)units in the input layer, 4 units in the hidden layer and 2 units in the output layer (3-4-2)

The multi-layer feed-forward network imposes no restrictions on the number of units in the input, hidden and output layers. This kind of network is closely related to some types of regression, hence similarities can be investigated examining the functional expression of a simple network (Haykin 1994; Bishop 1995). For instance, for the 3-4-2 network shown in Figure 1(b), the output, $a$, of the first hidden unit, 1, is obtained by first forming a weighted linear combination of the 3 input values, $\left(x_{1}, x_{2}, x_{3}\right)$, to give:

$$
s_{1}=w_{11} x_{1}+w_{12} x_{2}+w_{13} x_{3}+w_{10}=\sum_{i=0}^{3} w_{1 i} x_{i}
$$

where $w_{12}$ denotes the weight going from input unit 2 to hidden unit 1 , and $w_{10}$ is a required additional weight called bias. Then this linear sum is transformed using an activation function $g($.$) (usually g$ is a sigmoidal function such as the logistic function $g(s)=1 /\left(1+e^{-s}\right)$ or the hyperbolic tangent function $\left.g(s)=\left(e^{2 s}-1\right) /\left(e^{2 s}+1\right)\right)$ to give:

$$
a_{1}=g\left(s_{1}\right)
$$

Subsequently, the output of the net is obtained by transforming the output produced by the hidden units using a second layer of units. Thus, the explicit expression of the function represented by a 3-4-2 network can be written in the form (Haykin 1994): 


$$
\text { Output }_{k}=g\left(s_{k}\right)=g\left(\sum_{j=0}^{4} w_{k j}^{(2)} a_{j}\right)=g\left(\sum_{j=0}^{4} w_{k j}^{(2)} g\left(\sum_{i=0}^{3} w_{j i}^{(1)} x_{i}\right)\right)
$$

where the superscript of the weights denotes a weight coming from the first or second layer, and $k=1,2$ denotes output units 1 and 2 . In this simple network an input to the network will be passed through 20 weights, and modified by 6 non-linear functions. The function represented by this net could be thought of as a surface so that each peak or depression is created adding up small 'bumps' generated by the non-linear functions of the units (Bishop 1995). It has been demonstrated by several authors that adding enough units any continuous function could be represented by this method (i.e. universal approximation).

Implementing an ANN model usually involves several phases: data pre-processing, input selection, architecture selection, training, and independent performance assessment or validation. First, data are customarily filtered (e.g. missing records) and transformed, for instance, to match the range of the activation function or the Gaussian distribution. Note that ANNs are data-driven modelling tools, i.e. they learn from example. Consequently, training (sample) data must be representative of the population. Adequate model inputs are selected using numerical methods or a priori knowledge on the process being investigated. The appropriate network model and architecture are then selected. This is normally carried out by training different networks on a subset of the training dataset and assessing their generalisation performance on the unused training subset (validation subset) presenting it to the net with no learning taking place. The network having the lowest error is selected and the training parameters recorded. The selected model is then trained on the complete training dataset taking into account the recorded training parameters and its overall generalisation performance is assessed using an independent and previously withheld data set.

\section{SPATIAL INTERPOLATION USING ANN}

ANNs have been successfully used both for classification and regression in environmental applications. Time series forecasting has particularly benefited from the use of ANN models. Spatial interpolation of environmental data is related to forecasting in the sense that close observations (in this case in space) are correlated. Thus, as in forecasting, (spatially) lagged observations can be used as inputs to the model. Field variables such as natural radiation usually show a spatial correlation. Hence incorporating neighbour observations to spatial regression models based on spatial coordinates may improve estimation performance. In contrast to other techniques, ANN models can use geographical covariates, surrounding observations (local) in space or/and time, and available qualitative information about the process as inputs in order to estimate the output variable (e.g. natural radiation) (Figure 2). We apply here this technique to estimate daily natural radiation level and distribution for one day using prior information. 


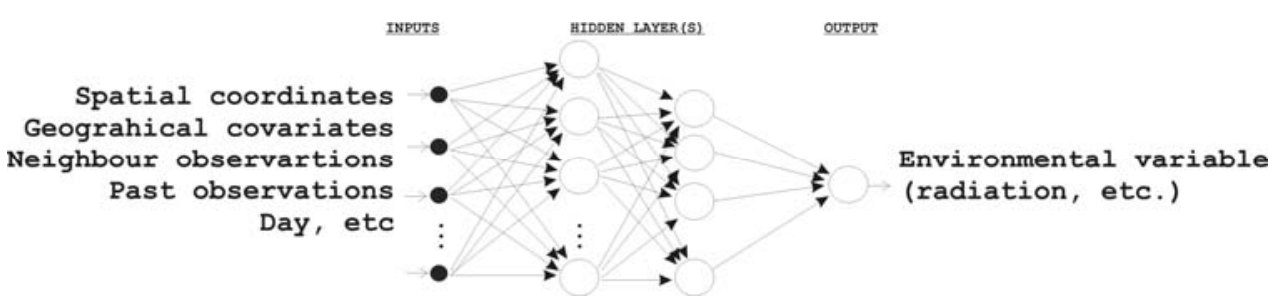

Figure 2

Schematic of ANN model for spatial estimation of environmental data

In this study, we use feed-forward back-propagation networks trained with the Resilient propagation algorithm for automatic mapping of daily measurements of natural radiation. Networks were trained to learn: (a) the relationship between daily measurements and their spatial coordinates, and (b) the relationship between daily measurements made at one site and information from a number of surrounding sites (measurement and distance). Network development was performed using the Stuttgart Neural Network Simulator, SNNS, software (Zell et al. 1998). Here, both model architecture selection and training were performed in a single step using a genetic algorithm provided with SNNS. In the estimation stage, the trained model was presented with output patterns with no learning taking place to generate estimations of radiation level for a particular location and date.

Uncertainty associated with the estimations was calculated repeating the training procedure 6 times starting from different random seeds. Estimation variance associated to each site was assimilated to uncertainty level for the site.

\section{USE OF PRIOR INFORMATION}

Network model development was carried out using available prior information corresponding to the 10-day series of natural background radiation measurements. Inputs to the model included spatial coordinates (eating and northing) of the reference site, and information corresponding to the six closest neighbour observations surrounding each reference site. Geostatistical variographic analysis of prior data indicated that trend surface residuals of mean radiation level for the ten-day series at a site was on average spatially correlated with up to the 16th surrounding closest sites. However, in order to simplify model development and model complexity only the six closest sites to each reference point were selected for the analysis. It was assumed that including spatial coordinates as inputs might account for a potential trend, while including neighbour observations might account for local spatial autocorrelation. Neighbour site data included radiation level for the corresponding day and distance vector. Distance vector (distance modulus and polar angle) was decomposed into two orthogonal components corresponding to easting and northing. Apart from radiation measurement, three distance parameters were eventually used as inputs for each neighbour: inverse of distance modulus, and inverse of easting-distance and northing-distance. This made a total of 26 input units (Figure 3). Network output consisted of the natural radiation observation for the reference site for the corresponding day. This made a single output unit. Hence, target values consisted of observations made at a site for a particular day of the prior 10-day series. 


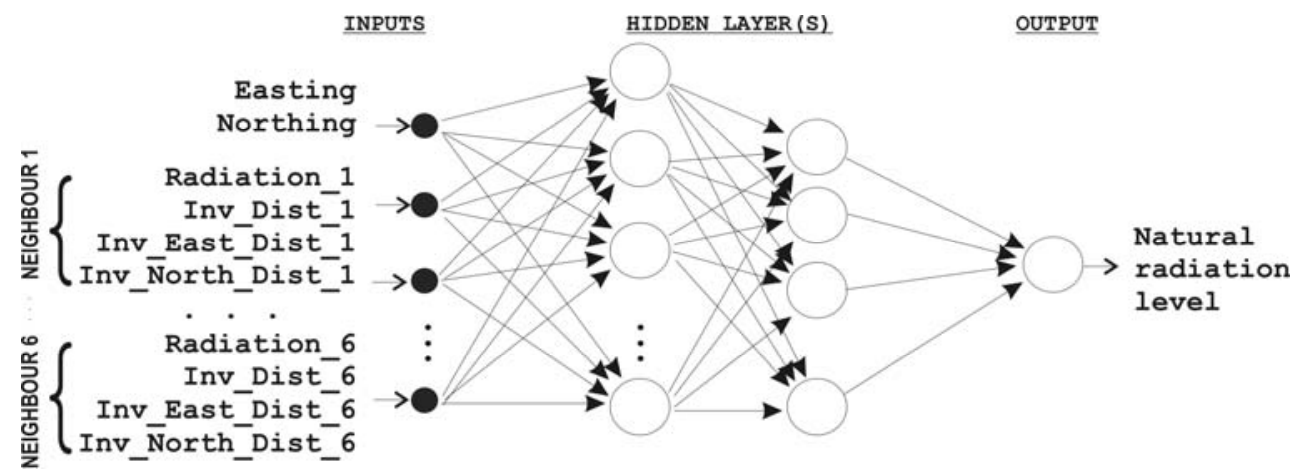

Figure 3

Inputs $(26=2+6 \times 4)$ and output (1) of ANN model for spatial estimation of natural radiation levels

The procedure above resulted in 2,000 training patterns or cases (200 sites by 10-day series of measurements). Available patterns were randomly divided into a training subset $(95 \%)$ and a validation subset (5\%). This corresponded to 1905 and 95 patterns, respectively. The validation subset was used for generalization assessment. A small validation set was generated to use as many available patterns as possible for network training.

Patterns for the estimation stage were constructed following the same process but using measurements at the 200 prior sites for the 11 th day of the series, i.e. the day for which estimations had to be generated, as neighbour sites, and the 808 additional test sites as the reference sites. The model trained on the first 10 days was used to estimate the 808 values of the 11 th day, using the 200 radiation values of the 11 th day only for neighbourhood calculations. Thus, patterns for the estimation stage were presented to the trained network with no learning taking place to produce estimations at the 808 unsampled locations for day 11 th. The procedure was repeated for the joker dataset.

\section{TUNING THE ALGORITHMS}

Several techniques have been devised for ANN model selection and tuning. A widely used approach for model selection involves training all possible network architectures using cross-validation (e.g. leave-on-out, k-fold) and early stopping. Other approaches use search heuristics based on evolutionary or genetic algorithms. An evolutionary approach was chosen for this study.

Network architecture and parameters were selected using the ENZO genetic algorithm (Braun and Ragg 1995). ENZO uses the paradigm of evolution for optimizing the topology (number of units, connectivity of units) and the paradigm of learning for optimizing the coefficients (weights and thresholds). It evolves a population of networks by generating offsprings through mutating the topology of the parent network and by optimizing the coefficients with a gradient descent algorithm. A three-layer parent network with 26 input units, 50 hidden units, and 1 output unit was used. The Resilient propagation with adaptive weight-decay (RpropMAP) training algorithm was selected (Bishop 1995; Zell et al. 1998). Training algorithm parameters were set to small initial values. These parameters were updated every 200 training epochs. The activation function was taken to be the logistic function for all units. Available data were linearly rescaled to the range $(0,1)$, to match the range of the activation function and to prevent from slow network convergence during the training phase due to disparate variable 
ranges. Minimum and maximum values used for rescaling radiation levels were set to values slightly smaller than the minimum value $(54.4 \mathrm{nSv} / \mathrm{h})$ and slightly larger than the maximum value $(157.0 \mathrm{nSv} / \mathrm{h})$, respectively, of the prior 10 -day series data. Thus maximum and minimum values were set to 50.0 and $160.0 \mathrm{nSv} / \mathrm{h}$, respectively. The error function being minimised was least squares. Training was stopped if the mean training error was smaller than a threshold value (0.0005) or the number of training epochs reached a maximum value of 6,000. Criteria used for measuring the performance (i.e. fitness) included error on training set, generalisation capability error on the validation set, number of units, and number of weights. Thus, networks with small training and validation errors along with small numbers of units and weights were preferred to the rest of networks of the population being evolved. Scheme used to weight relative importance of each performance criterion gave the larger weight to the generalisation error (one order of magnitude larger than the rest).

\section{RESULTS}

Model development procedure generated a partially connected trained network with a single hidden layer with 13 units (26-13-1), and with 293 links. Estimations for the first and second datasets are presented below.

\section{OVERALL RESULTS}

Table 1 shows the minimum, maximum, mean, median and standard deviation of the 808 estimated values and observed values for the two datasets. Results for the first dataset suggest that the model underestimated observed values. In contrast, standard deviation of estimations was larger than standard deviation of true values. For the second dataset, results suggest that the model clearly failed to estimate extreme values. Maximum estimated value was one order of magnitude smaller than the true observed maximum value. Mean, median and standard deviation were also underestimated.

\begin{tabular}{|l|r|r|r|r|r|}
\hline N = 808 & \multicolumn{1}{|c|}{ Min. } & \multicolumn{1}{l|}{ Max. } & \multicolumn{1}{l|}{ Mean } & \multicolumn{1}{c|}{ Median } & \multicolumn{1}{c|}{ Std. Dev. } \\
\hline Observed (first data set) & 57.0 & 180.0 & 98.0 & 98.8 & 20.0 \\
Estimates (first data set) & 50.3 & 154.0 & 96.4 & 96.8 & 23.3 \\
\hline Observed (second data set) & 57.0 & 1528.2 & 105.4 & 99.0 & 83.7 \\
Estimates (second data set) & 50.0 & 154.3 & 94.4 & 95.4 & 24.8 \\
\hline
\end{tabular}

Table 1

Comparison of the estimated and measured values (nSv/h)

Table 2 shows the mean absolute error (MAE), the bias (or mean error ME), and the root mean squared error (RMSE) of the predictions at the $\mathrm{n}=808$ locations for the two datasets. These are defined as:

$$
\begin{aligned}
M A E & =\frac{1}{n} \sum_{i=1}^{n}\left|a_{i}^{*}-a_{i}\right| \\
M E & =\frac{1}{n} \sum_{i=1}^{n}\left(a_{i}^{*}-a_{i}\right) \\
R M S E & =\sqrt{\frac{1}{n} \sum_{i=1}^{n}\left(a_{i}^{*}-a_{i}\right)^{2}}
\end{aligned}
$$


where $a^{*}$ is the estimated value at location $i$ and where $a_{i}$ is the true value. Pearson's $r$ coefficient of correlation between the estimated and true values is also given.

MAE, ME and RMSE for the first dataset indicated that despite error values were moderate, the model was not able to accurately estimate true values. Error statistics also show that the network model underestimated observed values. Pearson's $r$ indicated that correlation was also moderate. In contrast, MAE, ME and RMSE for the second dataset presented very large values, indicating that the model completely failed to estimate true values, especially outliers. In this case, Pearson's $r$ was almost 0 indicating that there was no correlation.

\begin{tabular}{|l|r|r|r|r|}
\hline Data sets & \multicolumn{1}{|l|}{ MAE } & \multicolumn{1}{l|}{ ME } & Pearson's $r$ & \multicolumn{1}{l|}{ RMSE } \\
\hline First data set & 16.0 & -1.7 & 0.55 & 20.8 \\
Second data set & 25.3 & -11.1 & 0.02 & 87.5 \\
\hline
\end{tabular}

Comparison of the errors
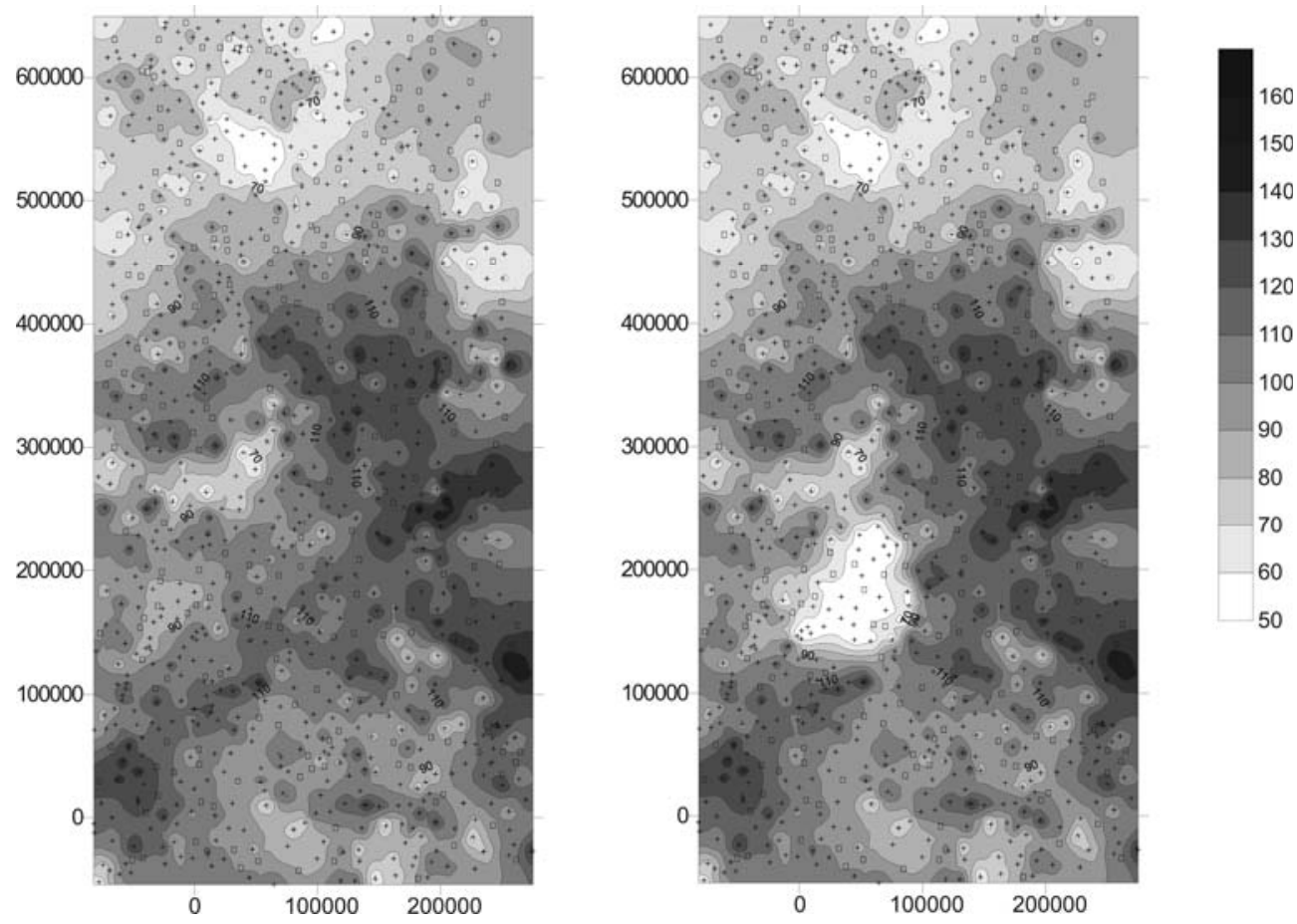

Figure 4

Isoline levels (nSv/h) for the 1st set (let) and the 2nd set (right). Crosses indicate locations of the estimated values. Empty squares indicate locations of the input values.

Maps with isolines showing the estimated values are presented in Figure 4. Spatial distribution of estimated values for the first dataset indicates that large natural background radiation levels concentrate in the central and South-Western sectors of the study area. Excluding the South-Western corner of the monitored area, the map showing the estimated values for the second dataset presents the same spatial distribution as the one 
for the first dataset. This map shows however that the network wrongly produced the smallest estimations for the expected large extreme values located in the area where accidental release of radioactivity in the environment was simulated.

Uncertainty associated to the estimations for the two datasets described above are shown as maps in Figure 5. For the first dataset, maximum uncertainty values tend to be located near the Eastern and Southern edges of the study area. Large uncertainties are also associated to estimations located at the central part of the Western edge of the study area. For the second dataset, massive uncertainties are associated to locations around the point where the accidental release of radioactivity in the environment was simulated. Networks generated in the six runs performed for uncertainty evaluation presented 13, 14 or 15 hidden layer units, with a comparable number of links.
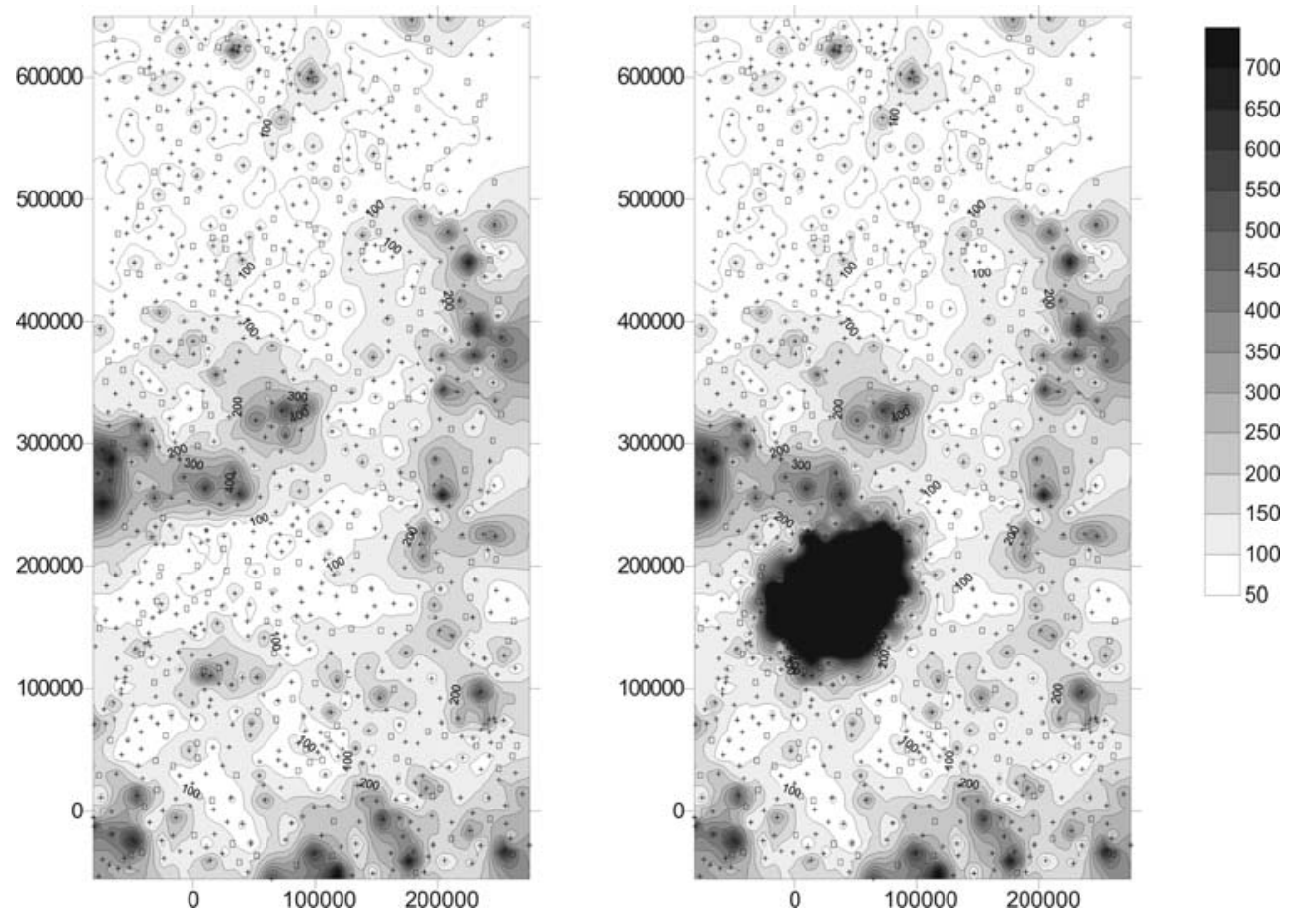

Figure 5

Isoline levels showing the uncertainty (estimation variance, $\left.(\mathrm{nSv} / \mathrm{h})^{2}\right)$ associated to the estimations obtained for the 1st set (let) and the 2nd set (right). Crosses indicate locations of the estimated values. Empty squares indicate locations of the input values.

A test using averaged results from the 6 training runs performed for uncertainty evaluation was carried out. As expected, using averaged estimations created by the 6 training experiments using different random seeds produced slightly better results for the first dataset than the results from a single network model presented above. Results for the second dataset were again clearly unacceptable. Tables 3 and 4 show statistics for the averaged estimations. Estimation and error statistics show that the model was also underestimating observed values. Maps of estimations with isolines are presented in Figure 6. 


\begin{tabular}{|l|r|r|r|r|r|}
\hline N = 808 & \multicolumn{1}{|c|}{ Min. } & \multicolumn{1}{|c|}{ Max. } & \multicolumn{1}{|c|}{ Mean } & \multicolumn{1}{c|}{ Median } & \multicolumn{1}{l|}{ Std. Dev. } \\
\hline Observed (first data set) & 57.0 & 180.0 & 98.0 & 98.8 & 20.0 \\
Estimates (first data set) & 56.5 & 139.4 & 96.9 & 98.6 & 18.7 \\
\hline Observed (second data set) & 57.0 & 1528.2 & 105.4 & 99.0 & 83.7 \\
Estimates (second data set) & 56.3 & 139.4 & 96.0 & 97.5 & 19.2 \\
\hline
\end{tabular}

Table 3

Comparison of the averaged estimated values from 6 runs and measured values $(\mathrm{nSv} / \mathrm{h})$

\begin{tabular}{|l|r|r|r|r|}
\hline Data sets & \multicolumn{1}{|l|}{ MAE } & \multicolumn{1}{l|}{ ME } & Pearson's $r$ & \multicolumn{1}{l|}{ RMSE } \\
\hline First data set & 12.1 & -1.2 & 0.67 & 15.8 \\
Second data set & 20.3 & -9.4 & 0.12 & 84.1 \\
\hline
\end{tabular}

Table 4

Comparison of the errors. Averaged results from 6 runs
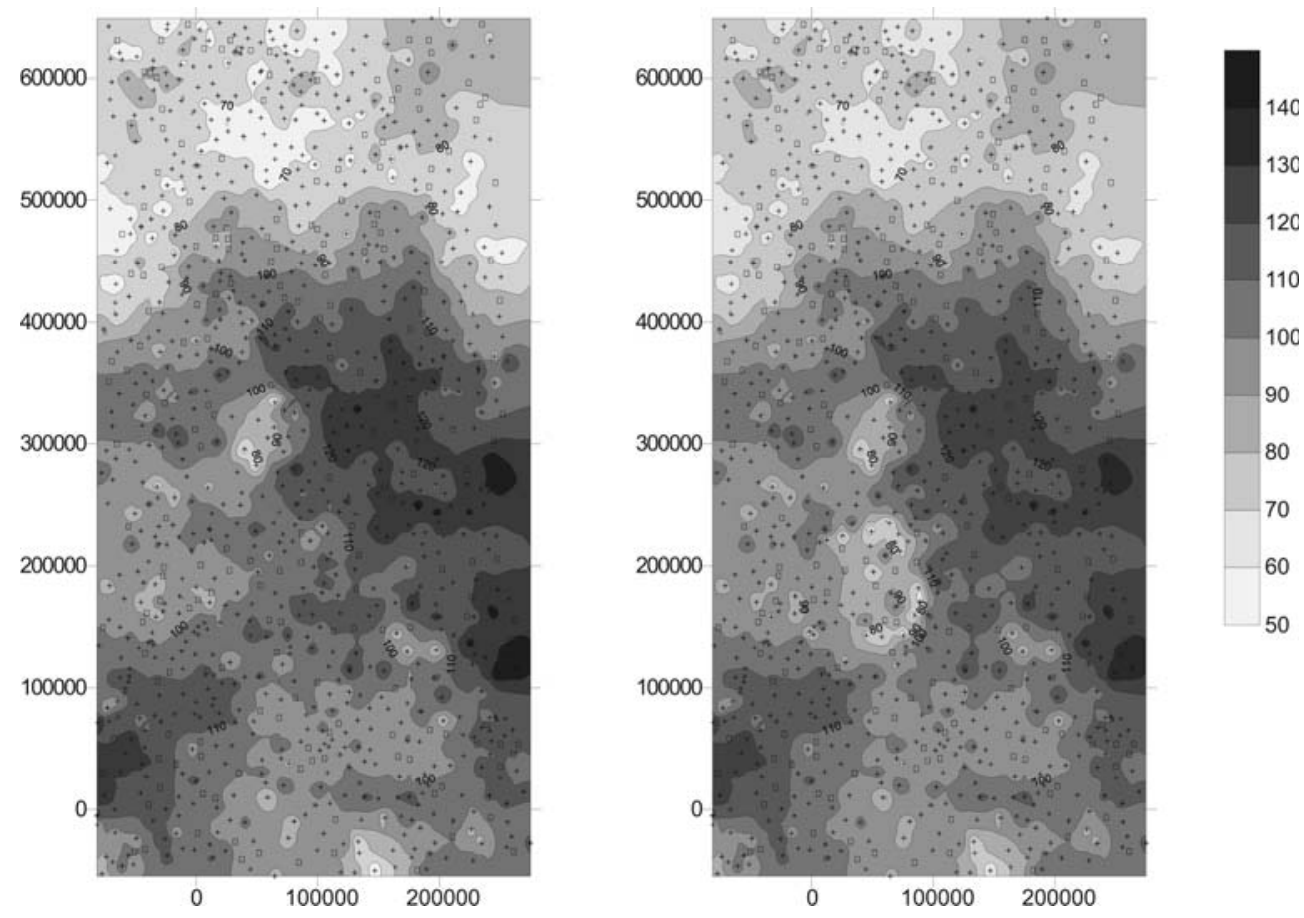

Figure 6

Averaged results from 6 training runs. Isoline levels (nSv/h) for the 1st set (let) and the 2nd set (right). Crosses indicate locations of the estimated values. Empty squares indicate locations of the input values.

\section{DETECTING ANOMALIES AND OUTLIERS}

As commented above, estimations for the second dataset including extreme values (joker dataset) were clearly unsatisfactory. The approach presented here proved to be useless for estimation of outliers. The trained network failed to estimate extreme values produced by the simulated accidental release of radioactivity in the South-Western corner of the monitored area. Figure 7 shows the estimates obtained for the 2 nd set in $3 \mathrm{D}$. It can be better seen that the trained network wrongly produced the lowest estimations for the extreme values. 


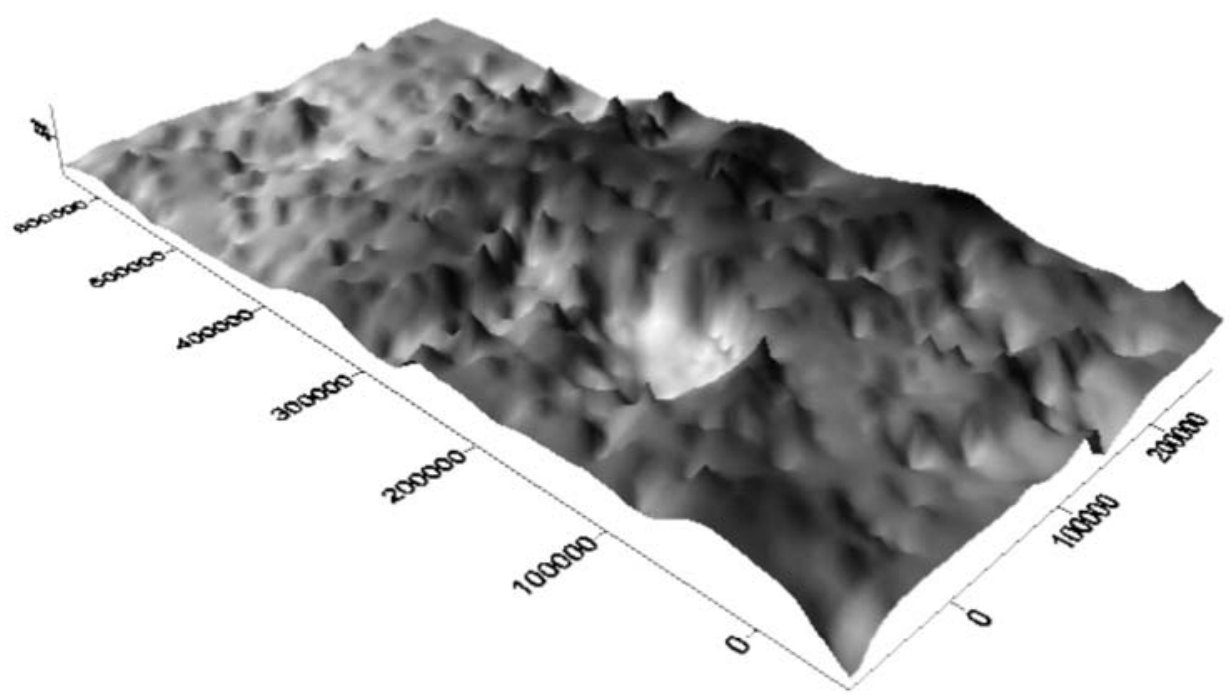

Figure 7

3D map showing extreme values found in the 2 nd set (vertical scale in $\mathrm{nSv} / \mathrm{h})$.

An additional experiment was performed (after true values were released) to test the ability of a network trained using a new dataset including outliers to estimate the second dataset. An accidental release of radioactivity to the environment was simulated using the expression used to create the second dataset (Dubois and Galmarini 2005). In this case the accidental release was simulated to take place at the North-Eastern corner (point 91, with spatial coordinates 16184,468883 ) in the 10 th day of the prior data series. Parameters used for the expression were:

- $\mathrm{Q}=1.510 \mathrm{e}+07$ [undefined units]

- $\mathrm{u}=7[\mathrm{~m} / \mathrm{s}]$

- $\mathrm{s}=6000[\mathrm{~m}]$

- $\mathrm{T}=20000[\mathrm{~s}]$

New input simulated radiation levels for the 10th day included now outliers (maximum value was $1862.6 \mathrm{nSv} / \mathrm{h}$ ). Radiation levels were transformed using the natural logarithm function. The same procedure described above was used for model development and training using this new training dataset. Results indicated that outliers were better detected. However, some false outliers were generated by the model. In addition, results for the first dataset were now unsatisfactory. Estimations for the first dataset also produced false outliers. Tables 5 and 6 show the comparison of basic statistics and errors. In this case, MAE, ME, and RMSE for the first dataset presented larger values than in previous experiments. ME presented now positive values indicating that the model overestimated observed values. Pearson's $r$ for the first dataset decreased to half of its value obtained in previous experiments. In contrast, Pearson's $r$ for the second dataset increased from 0.02 to $0.2 \mathrm{MAE}$ and RMSE for the second dataset were comparable to those obtained in previous experiments. Figure 8 shows the spatial distribution of 
estimations for the first and second datasets. It can be seen that extreme values are better detected, but now false potential outliers are introduced (Figure 9).

\begin{tabular}{|l|r|r|r|r|r|}
\hline $\mathbf{N}=\mathbf{8 0 8}$ & \multicolumn{1}{|c|}{ Min. } & \multicolumn{1}{|c|}{ Max. } & \multicolumn{1}{c|}{ Mean } & \multicolumn{1}{c|}{ Median } & \multicolumn{1}{c|}{ Std. Dev. } \\
\hline Observed (first data set) & 57.00 & 180.0 & 98.0 & 98.8 & 20.0 \\
Estimates (first data set) & 51.6 & 729.3 & 103.3 & 96.9 & 45.9 \\
\hline Observed (second data set) & 57.0 & 1528.2 & 105.4 & 99.0 & 83.7 \\
Estimates (second data set) & 51.6 & 1028.4 & 109.2 & 97.5 & 74.6 \\
\hline
\end{tabular}

Table 5

Comparison of the estimated and measured values (nSv/h). Model developed using simulated extreme values.

\begin{tabular}{|l|r|r|r|r|}
\hline Data sets & \multicolumn{1}{|l|}{ MAE } & \multicolumn{1}{l|}{ ME } & Pearson's $\boldsymbol{r}$ & \multicolumn{1}{l|}{ RMSE } \\
\hline First data set & 21.4 & 5.3 & 0.24 & 45.8 \\
Second data set & 30.5 & 3.8 & 0.20 & 96.6 \\
\hline
\end{tabular}

Table 6

Comparison of the errors. Network developed using training data including simulated extreme values.
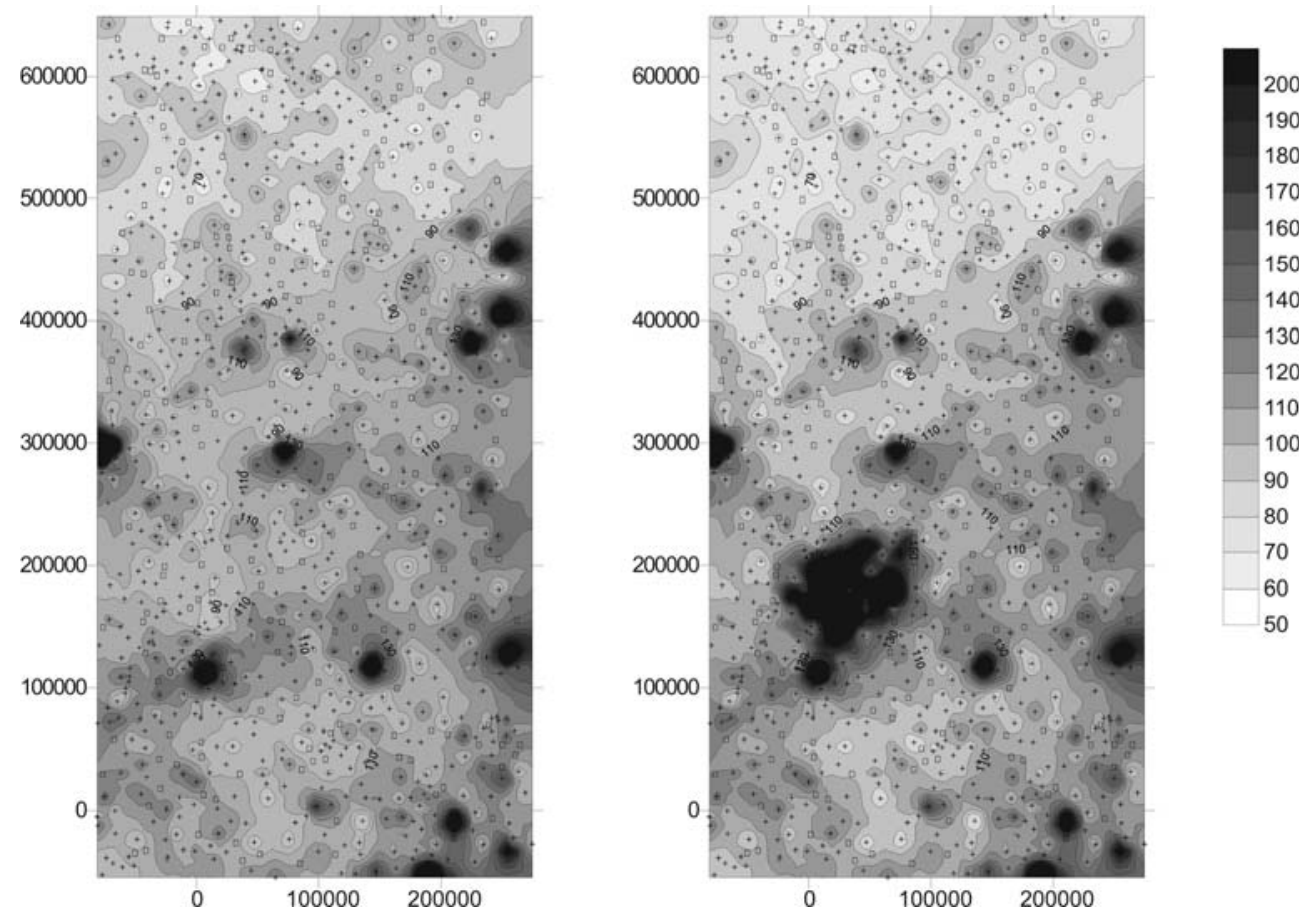

Figure 8

Results for network trained using data including simulated extreme values. Isoline levels (nSv/h) for the 1st set (let) and the 2nd set (right). Crosses indicate locations of the estimated values. Empty squares indicate locations of the input values. 


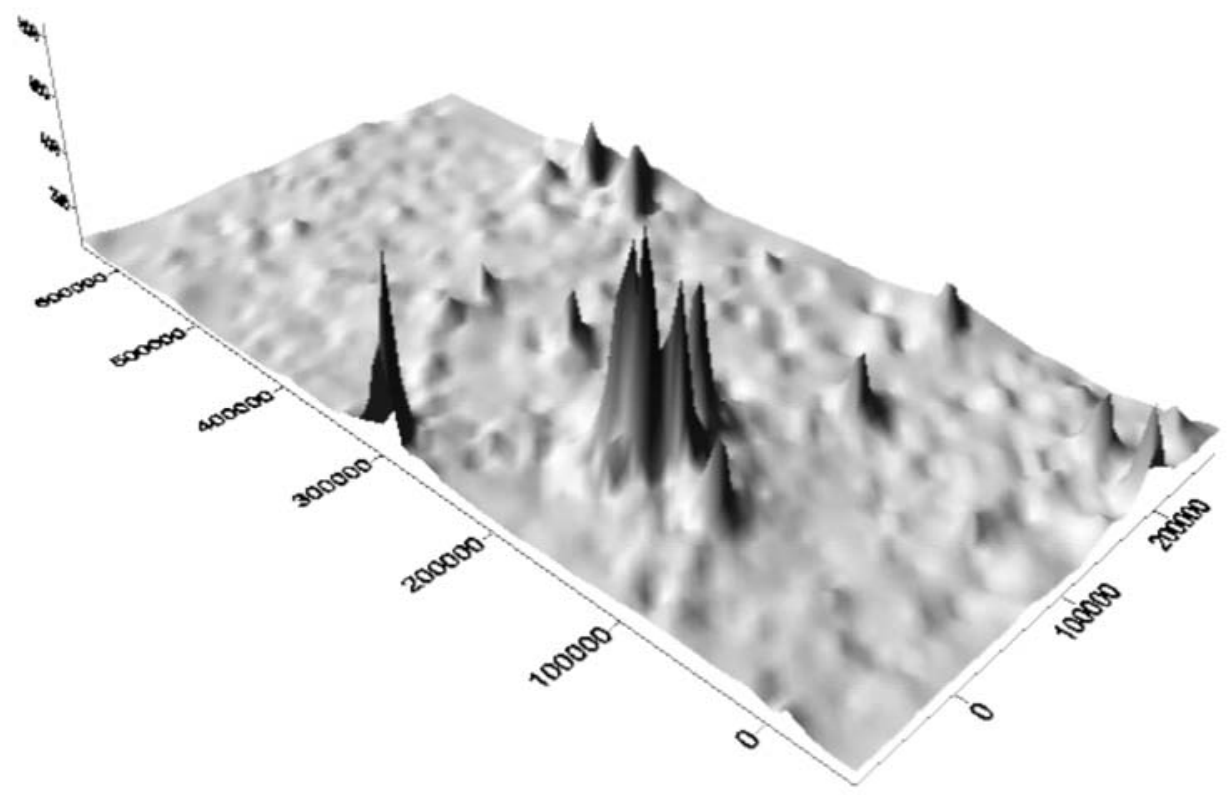

Figure 9

3D map showing extreme values found in the 2 nd set (vertical scale in $\mathrm{nSv} / \mathrm{h}$ ) generated with model developed using training data including simulated extreme values.

\section{DISCUSSION}

Results obtained in this study indicate that reasonably accurate estimations of natural background radiation levels could be obtained with a network trained using (background) prior data. On the other hand, estimation of data including extreme values proved to be a difficult task. In the automatic mapping exercise the network model was never presented with outlier values during the training stage. The net was trained using data in the range 54.4 to $157.0 \mathrm{nSv} / \mathrm{h}$, which were previously rescaled using minimum and maximum values of 50.0 and $160.0 \mathrm{nSv} / \mathrm{h}$, respectively. Network output values were thus 'safely' bounded to this range because of the activation function selected for the output unit. Trained networks produced wrong results when presented with extreme data out of the range of the training data. A test performed using an output unit linear activation function after exercise true values were released indicated that slightly better results could have been obtained using this model (test results are not presented). Nevertheless, output values for the second dataset with outliers included large errors. It is important to mention that using a wider range for data rescaling and an unbounded activation function would produce output values out of the range of the training data (i.e. extrapolation). As stressed in previous sections, ANNs are data-driven models which are good at interpolating but not at extrapolating. This means that, in general, a trained network will produce reliable estimations only in a data range commensurate to the range of the (target) data used for training. Consequently, an ANN developed for estimating natural background radiation levels will generally not be able to correctly estimate extreme values if outliers were not used for training. 
Results of the experiment performed using a new training dataset including simulated extreme values indicate that outliers could be better detected if outliers were also considered in model development. However, extreme values were not accurately estimated in any case. In addition, this model produced false outliers both for the first (with no outliers) and the second dataset. These results suggest that a single model for estimation of both background values and outliers might not be appropriate because performing well for one task implied performing badly for the other one. Thus, an approach using two different models for each purpose might be more successful. For instance, a model might be developed for estimating background values and another one for estimating extreme values. These two different models could be used in routine and emergency conditions, respectively. However, this approach poses a new problem since to launch the "emergency mapping algorithm" a signal must be triggered by the routine mapping system.

Model development (architecture selection and training) required several hours of computational time ( 6 to 7 hours). Once models were determined the estimation phase required an average computational time of 1.5 seconds for each dataset.

\section{CONCLUSIONS}

ANNs can be used for automatic mapping of environmental (background) data with moderate success. ANN models for spatial interpolation can incorporate prior information into the estimation process. However, network models developed using prior background radiation data presented large errors when estimating extreme values (extrapolation). The ability to interpolate accurately depends ultimately on the availability of data commensurate with the particular target scale of output. Consequently, network models trained for estimating background values will usually not produce adequate results for extreme values. On the other hand, network models trained to estimate both background and extreme values tend to produce false outliers.

The ANN approach presented here to automatic mapping of environmental data was clearly inappropriate for dealing with outliers. Results obtained suggest that developing two different models for estimating background values and extreme values, respectively, might be a potentially more successful approach. This however introduces new operational problems. This approach would need further investigation.

\section{ACKNOWLEDGMENTS}

This paper was improved by the comments of two anonymous referees.

\section{REFERENCES}

Antonic, O.; Krizan, J.; Marki, A.; Bukovec, D. 'Spatio-temporal interpolation of climatic variables over large region of complex terrain using neural networks'. Ecological Modelling 2001; 138(1-3): 255-263.

Bishop, C. M. Neural Networks for Pattern Recognition. Oxford: Clarendon Press; 1995.

Braun, H.; Ragg, T. ENZO - Evolution of neural networks. University of Karlsruhe, Germany: Institute for Logic, Complexity and Deduction Systems; 1995. URL: http://www-ra.informatik.uni-tuebingen.de/downloads/SNNS/ 
Bryan, B. A.; Adams, J. M. 'Three-dimensional neurointerpolation of annual mean precipitation and temperature surfaces for China'. Geographical Analysis 2002; 34(2): 93-111.

De Bollivier, M.; Dubois, G.; Maignan, M.; Kanevsky, M. 'Modified multilayer perceptron with local constraint: Artificial Neural Networks as an emerging method in spatial data analysis'. Nuclear Instruments and Methods in Physics Research 1997; A 389: 226-229.

Demyanov, V.; Soltani, S.; Kanevski, M.; Canu, S.; Maignan, M.; Savelieva, E.; Timonin, V.; Pisarenko, V. 'Wavelet analysis residual kriging vs. neural network residual kriging'. Stochastic Environmental Research and Risk Assessment 2001; 15(1): 18-32.

Dubois, G.; Shibli, S. A. R. 'Monitoring of environmental radioactivity: automatic mapping or expert-dependant systems?’. In: Dubois, G.; Malczewski, J.; De Cort, M., editors. Mapping radioactivity in the environment - Spatial Interpolation Comparison 97. Brussels: European Comision, EUR 20667 EN; 2003. pp. 253-268.

Dubois, G.; Galmarini, S. 'Introduction to the Spatial Interpolation Comparison (SIC) 2004 exercise and presentation of the data sets'. Applied GIS; 1(2): 09-01 to 09-11.

Haykin, S. Neural Networks, a comprehensive Foundation. New York: Macmillan College Publishing Company; 1994.

Holdaway, M. R. 'Spatial modelling and interpolation of monthly temperature using kriging'. Climate Research 1996; 6: 215-225.

Hutchinson, M. F. 'Interpolating mean rainfall using thin plate smoothing splines'. International Journal of Geographical Information Systems 1995; 9(4): 385-403.

Luk, K. C.; Ball, J. E.; Sharma, A. 'A study of optimal model lag and spatial inputs to artificial neural network for rainfall forecasting'. Journal of Hydrology 2000; 227(1-4): 56-65.

Pariente, D.; Laurini, R. 'Statistical spatio-temporal data interpolation and extrapolation based on neural networks'. Proceedings of Workshop on New Tools for Spatial Analysis; Lisbon; 1993.

Pariente, D. 'Geographic interpolation and extrapolation by means of neural networks'. Proceedings EGIS/MARI '94, Fifth European Conference and Exhibition on Geographical Information Systems EGIS. Paris; 1994.

Rigol, J. P.; Jarvis, C. H.; Stuart, N. 'Artificial neural networks as a tool for spatial interpolation'. International Journal of Geographical Information Science 2001; 15(4): 323-343.

Rizzo, D. M.; Dougherty, D. E. 'Characterization of aquifer properties using artificial neural networks - neural kriging'. Water Resources Research 1994; 30(2): 483-497.

Rumelhart, D. E.; Hinton, G. E.; Williams, R. J. 'Learning representations by back-propagating errors'. Nature 1986; 323: 533-536.

Sarle, W. S. Comp.ai.neural-nets News Group Archive. 1999. URL: ftp://ftp.sas.com/pub/neural/FAQ.html

Snell, S. E.; Gopal, S.; Kaufmann, R. K. 'Spatial interpolation of surface air temperatures using artificial neural networks: Evaluating their use for downscaling GCMs'. Journal of Climate 2000; 13(5): 886-895.

Zell, A.; Mamier, G.; Vogt, M.; Mache, N.; Hübner, R.; Doring, S.; Hermann, K.-U.; Soyez, T.; Schmalzl, M.; Sommer, T.; Hatzigeorgiou, A.; Posselt, D.; Schreiner, T.; Kett, B.; Clemente, G.; Wieland, J. Stuttgart Neural Network Simulator software user manual. Germany: University of Stuttgart; 1998. URL:

http://www.informatik.uni-tuebingen.de/SNNSinfo /UserManual/UserManual.html

Cite this article as: Rigol-Sanchez, J. P. 'Spatial interpolation of natural radiation levels with prior information using back-propagation artificial neural networks'. Applied GIS, Vol 1, No 2, 2005. pp. 18-01 to $18-15$. DOI: $10.2104 / \mathrm{ha0} 40018$

Copyright $\odot 2005$ J. P. Rigol-Sanchez 